\title{
Associações entre prática de atividade física e qualidade do sono no contexto pandêmico de distanciamento social
}

\author{
Associations between physical activity and sleep quality in the pandemic context of social distancing \\ Asociaciones entre actividad física y calidad del sueño en el contexto pandémico de distancia social
}

Recebido: 23/12/2020 | Revisado: 31/12/2020 | Aceito: 31/12/2020 | Publicado: 03/01/2021

\author{
Marcos Venício Cavalcante \\ ORCID: https://orcid.org/0000-0003-1087-6922 \\ Instituto Federal de Educação, Ciência e Tecnologia do Ceará, Brasil \\ E-mail: marcosvenicio@gmail.com \\ Rômmulo Celly Lima Siqueira \\ ORCID: https://orcid.org/0000-0003-0533-9305 \\ Instituto Federal de Educação, Ciência e Tecnologia do Ceará, Brasil \\ E-mail: rommulo.siqueira@ifce.edu.br \\ Raynara Correia Costa \\ ORCID: https://orcid.org/0000-0002-2742-5522 \\ Instituto Federal de Educação, Ciência e Tecnologia do Ceará, Brasil \\ E-mail: raynara.correiacosta@gmail.com \\ Thamirys Freitas Lima \\ ORCID: https://orcid.org/0000-0001-7875-6519 \\ Instituto Federal de Educação, Ciência e Tecnologia do Ceará, Brasil \\ E-mail: thamiryslima1@gmail.com \\ Tiago Maia Costa \\ ORCID: https://orcid.org/0000-0001-5074-7434 \\ Instituto Federal de Educação, Ciência e Tecnologia do Ceará, Brasil \\ E-mail: tiago.maia@ifce.edu.br \\ Cicero Luciano Alves Costa \\ ORCID: https://orcid.org/0000-0002-4428-5111 \\ Instituto Federal de Educação, Ciência e Tecnologia do Ceará, Brasil \\ E-mail: cicero.luciano-costa@ifce.edu.br
}

\begin{abstract}
Resumo
Este estudo teve como objetivo investigar possíveis associações entre prática de atividade física e qualidade do sono durante o período de distanciamento social da pandemia de COVID-19. Participaram 458 adultos com idades entre 18 e 59 anos. O nível de atividade física foi investigado por meio do Questionário Internacional de Atividade Física (IPAQ) e a qualidade do sono pelo Mini Sleep Questionnaire (MSQ). Os resultados demonstraram que 79,3\% dos participantes com algum nível de dificuldade do sono. A prática de atividade física demonstrou associação com a qualidade do sono $(\mathrm{OR}=1,922, \mathrm{IC}=1,121-3,295)$. A ANOVA oneway apontou diferença significativa na qualidade do sono entre os diferentes níveis de atividade física. O nível muito ativo apresentou melhor qualidade do sono em comparação a ativos, insuficientemente ativos e sedentários $(\mathrm{p}<0,05)$. Observou-se correlação negativa e significativa $(\mathrm{r}=-0,187, \mathrm{p}=0,001)$ entre o tempo gasto em atividades físicas vigorosas e o somatório do questionário de qualidade do sono. Não foram encontradas relações significativas entre qualidade do sono e atividade física moderada e/ou caminhada. Conclui-se que durante o período pandêmico, a prática de atividade física de característica vigorosa pode contribuir para uma melhor qualidade do sono. Recorrendo a uma das hipóteses que procuram explicar a relação entre exercício físico e sono, infere-se que este achado pode ser explicado pela sensação de fadiga decorrente do maior dispêndio energético proporcionado por estas atividades.
\end{abstract}

Palavras-chave: COVID-19; Atividade física; Qualidade do sono; Dispêndio energético.

\begin{abstract}
This study aimed to investigate possible associations between physical activity and sleep quality during social distance in the COVID-19 pandemic. Four hundred and fifty-eight adults aged between 18 and 59 years participated. The level of physical activity was investigated using the International Physical Activity Questionnaire (IPAQ) and the quality of sleep by the Mini Sleep Questionnaire (MSQ). The results showed that $79.3 \%$ of the participants had some level of sleep difficulty. The practice of physical activity demonstrated association with sleep quality $(\mathrm{OR}=1.922$, CI = 1.121-3.295). The oneway ANOVA showed significant difference in the sleep quality between the different physical activity levels. The very active level showed better quality of sleep compared to active, insufficiently active and sedentary $(p<0.05)$. There was a negative and significant correlation $(r=-0.187, p=0.001)$ between the time spent in vigorous physical activities and the sum of the sleep quality questionnaire. No significant relationship was found between sleep quality and moderate physical activity and/or walking. It is concluded that during the pandemic
\end{abstract}


period, the practice of vigorous physical activity can contribute to better sleep quality. Using one of the hypotheses that seek to explain the relationship between physical exercise and sleep, it is inferred that this finding can be explained by the feeling of fatigue resulting from the greater energy expenditure provided by these activities.

Keywords: COVID-19; Physical activity; Sleep quality; Energy expenditure.

\section{Resumen}

Este estudio tuvo como objetivo investigar las posibles asociaciones entre la actividad física y la calidad del sueño durante la distancia social en la pandemia de COVID-19. Participaron 458 adultos de entre 18 y 59 años. El nivel de actividad física se investigó mediante el Cuestionario Internacional de Actividad Física (IPAQ) y la calidad del sueño mediante el Mini Cuestionario de Sueño (MSQ). Los resultados mostraron que 79,3\% de los participantes tenían algún nivel de dificultad para dormir. La práctica de actividad física demostró asociación con la calidad del sueño (OR $=1,922$, IC $=1,121-3,295)$. El ANOVA de una vía mostró diferencia significativa en la calidad del sueño entre los diferentes niveles de actividad física. El nivel muy activo mostró una mejor calidad de sueño en comparación con el activo, insuficientemente activo y sedentario $(\mathrm{p}<0,05)$. Hubo una correlación negativa y significativa $(\mathrm{r}=-0,187, \mathrm{p}=$ 0,001) entre el tiempo dedicado a actividades físicas vigorosas y la suma del cuestionario de calidad del sueño. No se encontró relación significativa entre la calidad del sueño y la actividad física moderada y/o caminar. Se concluye que, durante el período pandémico, la práctica de actividad física vigorosa puede contribuir a una mejor calidad del sueño. Utilizando una de las hipótesis que buscan explicar la relación entre el ejercicio físico y el sueño, se infiere que este hallazgo puede explicarse por la sensación de fatiga resultante del mayor gasto energético que aportan estas actividades.

Palabras clave: COVID-19, Actividad física, Calidad del sueño; Gasto energético.

\section{Introdução}

Os primeiros relatos da COVID-19, doença causada pelo novo coronavírus Coronavírus-2, SARS-CoV-2 (Síndrome Respiratória Aguda Grave de Coronavírus 2) aconteceram no final do ano de 2019 na China, mais especificamente na província Hubei na cidade de Wuhan. Desde então, milhares de pessoas por todo o mundo já foram contaminadas e tantas outras foram mortas em decorrência de complicações causadas pelo novo vírus. De acordo com Pascarella et al. (2020) a infecção pelo novo coronavírus se assemelha bastante a já conhecida gripe, causando tosse e febre, entretanto, também se verificam a possibilidade de lesões pulmonares em indivíduos de todas as idades. No dia 11 de março de 2020, a Organização Mundial de Saúde (OMS) declarou que o surto do novo vírus se tratava de uma pandemia, fazendo com que os chefes de estado de todo o mundo tomassem medidas a fim de proteger a vida de seus cidadãos e evitar que a doença se alastrasse ainda mais.

Sem a existência de uma vacina para a COVID-19, diversas providências foram tomadas em busca de conter a rápida contaminação pela doença. Recomendou-se então o uso de máscaras, a lavagem adequada das mãos, a quarentena, o isolamento social e medidas de distanciamento social (Silveira, Carvalho, Seara \& Olivares, 2020). As medidas de distanciamento social buscam evitar o contato físico entre as pessoas para que o vírus não se multiplique. De acordo com Wilder-Smith e Freedman (2020)), o distanciamento social é utilizado em comunidades em que pode existir a transmissão comunitária da doença, não sabendo ao certo como foi adquirido. Quando o isolamento das pessoas infectadas não é considerado eficiente. Nesta situação, torna-se necessário que as pessoas respeitem a distância adequada entre elas e não se encontrem e/ou frequentem estabelecimentos em que haja potencial de aglomeração (Faro et al., 2020).

Considerando o atual contexto pandêmico que obrigou a instauração do distanciamento social, causando alterações na rotina das pessoas, modificando as rotinas trabalhistas e educacionais, parte da população tem se privado de sair de suas residências como precaução de um maior raio de contágio pelo vírus. Apesar de ser uma importante medida preventiva contra a propagação do vírus, o distanciamento tem provocado efeitos negativos em diferentes dimensões do comportamento das pessoas, causando alterações na saúde mental (Matias, Dominski, \& Marks, 2020; Ornell, Schuch, Sordi, \& Kessler, 2020), na alimentação (Ammar et al., 2020), no sono (Monteiro, Santos Neto \& Pires de Souza, 2020; Pinto et al., 2020) e na prática de atividade física (Costa, Costa, Barbosa Filho, Bandeira, \& Siqueira, 2020; Nyenhuis, Greiwe, Zeiger, Nanda, \& Cooke, 2020).

Como mencionado anteriormente, dentre as diversas mudanças ocorridas decorrentes do distanciamento social a 
prática de atividade física estava entre elas. Estudos como os de Giustino et al. (2020) e Costa et al. (2020) constataram que o nível de atividade física dos indivíduos participantes da pesquisa durante a pandemia diminuiu quando comparado com o período anterior. Esta queda pode estar relacionada ao fechamento das academias durante vários meses do período da pandemia, falta de um profissional especializado para prescrever e acompanhar o treino, ausência de material e espaço em casa para prática, dentre outros fatores.

Além da prática de atividade física, outro fator citado que sofre alterações durante o período de distanciamento social é o sono. Em um estudo realizado no Brasil por Bezerra, da Silva, Soares e da Silva (2020), a maioria dos participantes respondeu que tiveram mudanças no seu tempo normal de sono em relação a antes da pandemia, sendo que alguns obtiveram no período pandêmico mais horas de sono e outros menos. Ademais, Barros et al. (2020), também observaram que parte dos indivíduos que não apresentavam problemas no sono antes da pandemia passaram a tê-los, bem como os que já tinham, apresentaram um agravamento desse quadro durante o período de distanciamento social. Desta forma, é relevante buscar entender quais fatores poderiam contribuir para a diminuição dos prejuízos no sono causados pelo contexto da pandemia.

Um dos fatores que a literatura tem reportado como capaz de promover benefícios na qualidade do sono é a atividade física (Khan, Chu, Kirk, \& Veugelers, 2015; Wu et al., 2015). A prática de atividade física possui um efeito sincronizador indiretamente ao relógio biológico, causando assim uma melhor qualidade do sono nos praticantes (Mello et al., 2005; Ropke et al., 2018). Uma vez que tanto a prática de atividade física, como o sono foram afetados durante pandemia e sabendo que a atividade física traz benefícios à qualidade do sono, o presente estudo objetiva investigar possíveis associações entre prática de atividade física e qualidade do sono no contexto pandêmico de distanciamento social. Hipotetizamos que o maior nível de atividade física está associado com a melhor qualidade do sono.

\section{Metodologia}

Trata-se de uma pesquisa descritiva-correlacional, de abordagem quantitativa (Thomas, Nelson \& Silverman, 2012). Participaram do estudo 458 sujeitos adultos de ambos os sexos, sendo 266 mulheres (58,08\%) e 192 homens (41,92\%), com idades entre 18 e 59 anos (28,3+8,7 anos). Todos os participantes declararam a concordância em participar da pesquisa através do Termo de Consentimento Livre e Esclarecido (TCLE). Os procedimentos éticos preconizados pela Declaração de Helsinque foram seguidos e os protocolos de pesquisa foram aprovados pelo comitê de ética local (parecer $\mathrm{n}^{\circ}$ 4.050.668). A pesquisa foi realizada de forma online sendo disponibilizada e compartilhada através das redes sociais (WhatsApp, Instagram, Twitter e Facebook), com início às 13 horas e 16 minutos do dia 27 de julho de 2020 e sendo encerrada às 13 horas e 16 minutos do dia 4 de agosto de 2020. Durante este período o índice de isolamento social no Brasil variou de 37,4 \% a 39,2\%, segundo dados da empresa In Loco (2020). A pesquisa foi respondida por indivíduos de quase todos estados do Brasil, com exceção de Roraima.

Para a realização da pesquisa foi confeccionado um formulário no Formulários Google ${ }^{\circledR}$ composto por 4 partes. A primeira delas, trazia o Termo de Consentimento Live e Esclarecido - TCLE, o qual apresentava informações sobre a pesquisa e permitia ao voluntário escolher participar ou não da pesquisa. A segunda parte do formulário continha perguntas relacionadas aos aspectos sociodemográficos. Em seguida, o formulário trazia o Questionário Internacional de Atividade Física (IPAQ), versão curta. Este instrumento validado para brasileiros através dos pesquisadores do Centro de Estudos do Laboratório de Aptidão Física de São Caetano do Sul (CELAFISCS) (Matsudo et al., 2001) é um dos mais recomendados para investigar o nível de atividade física. O instrumento é dividido em quatro itens que investigam informações sobre o tempo e frequência semanal da prática de atividade física (vigorosa, moderada e caminhada), além do tempo em que o indivíduo fica sentado. O instrumento permite classificar o nível de atividade física em sedentário, insuficientemente ativo, ativo e muito ativo.

A última parte do formulário foi composta pela versão brasileira do Mini Sleep Questionnaire (MSQ), validado no Brasil por Falavigna et al. (2011), que tem como objetivo avaliar a qualidade do sono dos indivíduos através das respostas de 
10 questões de múltipla escolha. As questões contêm situações relacionadas aos distúrbios do sono, em uma escala de likert de 1 (Nunca) a 7 (Sempre). Através das somas destas respostas os participantes são classificados em relação ao nível de dificuldade do sono: com boa qualidade do sono (soma entre 10 a 24), dificuldade leve do sono (soma entre 25 a 27), dificuldade moderada do sono (soma entre 28 a 30) e dificuldade severa do sono (soma superior a 30) (Machado, 2017).

Os dados foram tabulados no pacote estatístico Statistical Package for the Social Sciences (SPSS® versão 20). A análise dos dados foi conduzida por meio de estatística descritiva e inferencial. Na análise descritiva utilizou-se medidas de média, desvio padrão e distribuição de frequência. Utilizou-se o teste Qui-quadrado para verificar associações entre as variáveis nível de atividade física e qualidade do sono em relação ao sexo e o Teste Z (com ajuste de Bonferroni) para comparação de proporções. O odds ratio com intervalo de confiança de $95 \%$ foi obtido para analisar a chance de ser suficientemente ativo influenciar na qualidade do sono. A homoscedasticidade de variância foi verificada pelo teste de Levene. O teste ANOVA Oneway foi utilizado para verificar diferenças do somatório do questionário do sono entre os participantes com diferentes níveis de atividade física. O post hoc de Tukey foi utilizado para verificar quais grupos se diferenciaram. Para verificar se alguma categoria de atividade física investigada pelo IPAQ se relaciona com a qualidade do sono foi aplicado o teste de correlação de Pearson. O nível de significância adotado para todos os testes foi de 5\%).

\section{Resultados}

A Figura 1 apresenta a distribuição de frequência do nível de atividade física dos indivíduos relacionada com o sexo. Nota-se que os participantes do sexo masculino apresentam um maior percentual de classificação em nível "muito ativo" em comparação ao sexo feminino. O teste Qui-quadrado apontou associação significativa entre sexo e nível de atividade física $(\mathrm{X} 2=10.752, \mathrm{p}=0,013)$ com sexo masculino apresentando uma maior proporção de nível muito ativo que o sexo feminino $(\mathrm{p}=0,001)$.

Figura 1 - Distribuição de frequência do nível de atividade física em função do sexo.

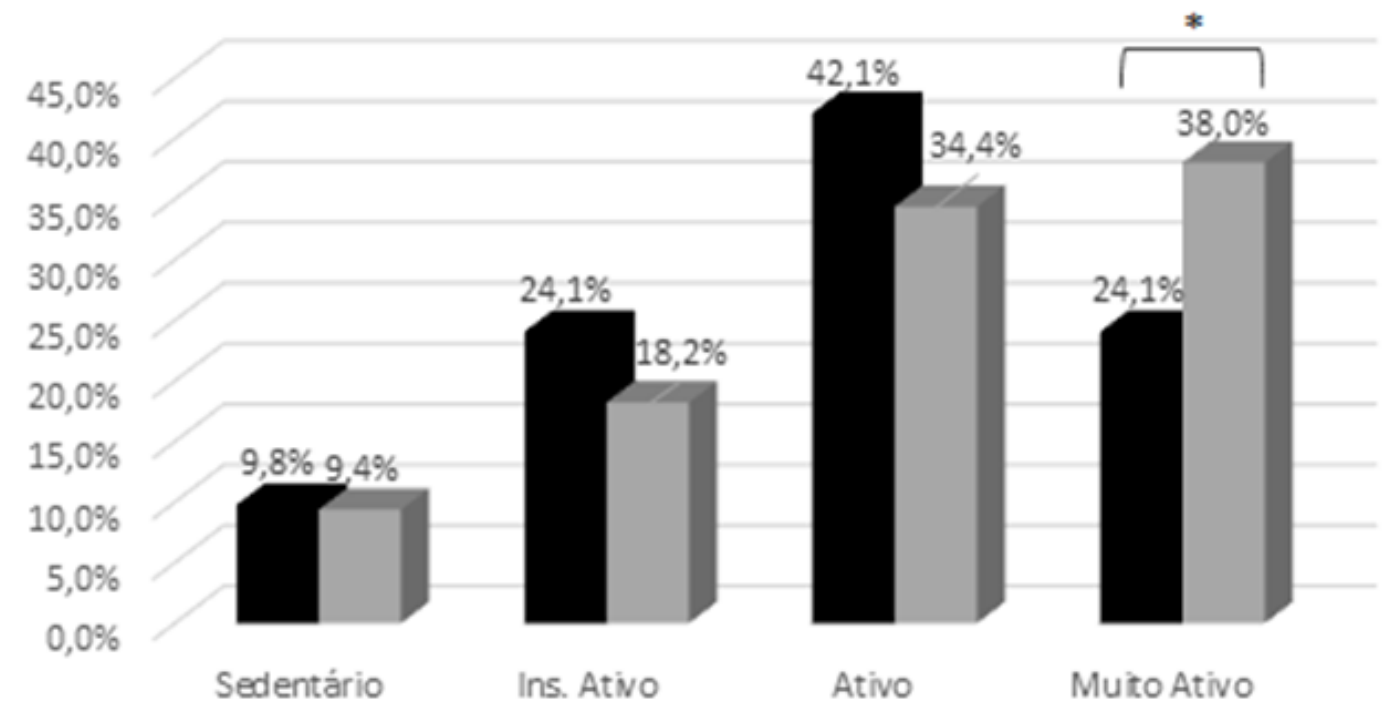

- Feminino Masculino

* p<0,05 - Qui-quadrado de associação. Fonte: Autores.

A Figura 2 apresenta a distribuição de frequência da classificação dos participantes em relação à qualidade do sono de 
acordo com o sexo. Nota-se que no período de distanciamento social mais da metade dos participantes estavam com dificuldade severa do sono, sendo que o sexo feminino apresentou um maior percentual nessa classificação com $57,5 \%$, enquanto o sexo masculino apresentou 51\% dos participantes com dificuldade severa do sono. No entanto, o teste Quiquadrado não apontou associação entre qualidade do sono e sexo (X2=2,039, p=0,564). No total, 79,3\% dos participantes apresentaram algum nível de dificuldade do sono.

Figura 2 - Distribuição de frequência do nível de dificuldade do sono em função do sexo.

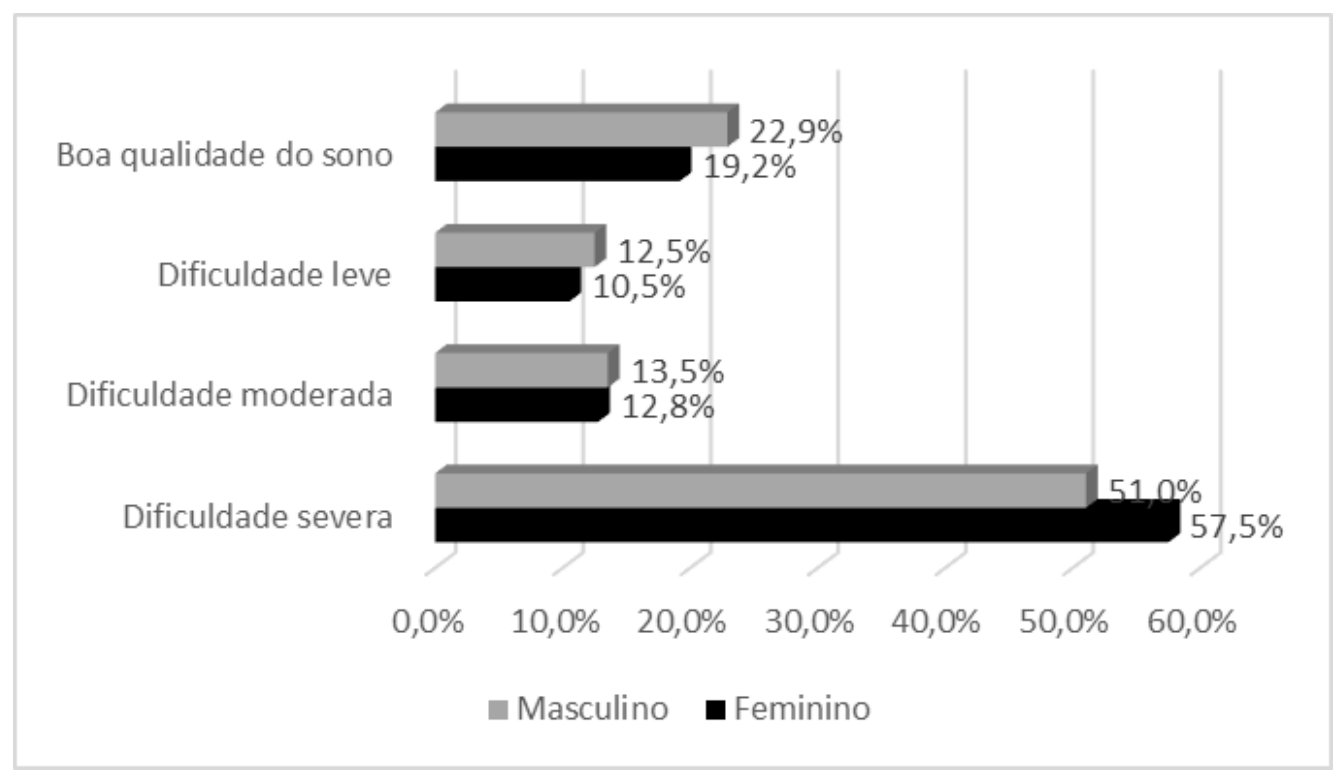

Fonte: Autores.

A Tabela 1 apresenta a distribuição de frequência e o odds ratio para a qualidade do sono em função do nível de atividade física. Para essa análise foram agrupados os participantes sedentários e insuficientemente ativos em um grupo e os ativos e muito ativos em outro. Para a variável dependente, os participantes com dificuldade leve, moderada e severa também foram agrupados. Embora a maioria dos participantes apresentem dificuldades no sono, percebe-se um maior percentual de participantes do grupo ativo/muito ativo com qualidade do sono. Esta associação foi apontada como significativa pelo teste Qui-quadrado $(\mathrm{X} 2=5,773, \mathrm{p}=0,016)$. Os resultados também demonstram que aqueles com maior nível de atividade física (Ativo/Muito Ativo) possuem maior chance de apresentarem boa qualidade do sono.

Tabela 1 - Distribuição de frequência, Odds Ratio e intervalo de confianças (IC) de 95\% da qualidade do sono em função do nível de atividade física.

Dificuldade do Sono

\section{Sedentário/Inativo}

\section{Ativo/Muito Ativo}

OR

IC $(95 \%)$
Qualidade do Sono

$$
123(86 \%)
$$

$$
240(76,2 \%)
$$

$$
1
$$

$$
75(23,8 \%)
$$

$(1,121-3,295)$

\footnotetext{
$* \mathrm{p}<0,05$ - Teste Qui-quadrado. Fonte: Autores.
} 
A Tabela 2 apresenta as médias e desvios-padrão do somatório do questionário de qualidade do sono em função dos diferentes níveis de atividade física. $\mathrm{O}$ teste ANOVA indicou diferenças significativas entre os grupos $(\mathrm{F}=7,344, \mathrm{p}=0,001)$. Na análise dos desdobramentos o post hoc de Tukey apontou diferenças entre os participantes classificados como muito ativos em comparação aos sedentários $(\mathrm{p}=0,005)$, insuficientemente ativos $(\mathrm{p}=0,003)$ e ativos $(\mathrm{p}=0,013)$, com média menos elevada para os participantes muito ativos.

Na análise da relação entre as categorias de atividade física do IPAQ e a qualidade do sono, o teste de correlação de Pearson apontou uma relação negativa e significativa entre o tempo gasto em atividades físicas vigorosas e o somatório do questionário de qualidade do sono $(\mathrm{r}=-0,187, \mathrm{p}=0,001)$. Para as atividades físicas moderadas $(\mathrm{r}=-0,073, \mathrm{p}=0,120)$ e caminhada ( $r=-0,041, p=0,382)$ não foram encontradas associações).

Tabela 2 - Média e desvio padrão do somatório do questionário para qualidade do sono.

\begin{tabular}{cccc}
\hline Nível de atividade Física & $\mathbf{N}$ & Média & Desvio Padrão \\
\hline Sedentário & 44 & $34,7^{\mathrm{a}}$ & 10,1 \\
Insuficientemente Ativo & 99 & $34,4^{\mathrm{b}}$ & 9,3 \\
Ativo & 178 & $32,6^{\mathrm{c}}$ & 8,6 \\
Muito Ativo & 137 & $29,6^{\mathrm{abc}}$ & 7,8 \\
\hline Total & 458 & 32,3 & 8,9 \\
\hline
\end{tabular}

$* \mathrm{p}<0,05$ - ANOVA Oneway. abc $\mathrm{p}<0,05$ - post hoc de Tukey. Fonte: Autores.

\section{Discussão}

Durante a pandemia do novo Coronavírus (SARS-CoV-2) e com o distanciamento social, estudos têm mostrado que ocorreram mudanças na prática de atividade física da população brasileira (Costa et al., 2020; Malta et al., 2020). Além disso, outro aspecto que apresentou mudanças nesse período foi o padrão de sono das pessoas, visto que fatores decorrentes da necessidade de distanciamento social parecem ter interferido na qualidade do sono (Absono, 2020; Pinto et al., 2020). Com isso, o presente estudo teve como objetivo investigar possíveis associações entre prática de atividade física e qualidade do sono no contexto pandêmico de distanciamento social. O nível de atividade física e a qualidade do sono foram investigadas através de questionários disponibilizados no Google Forms®, sendo divulgados por meio das redes sociais.

Em relação ao nível de atividade física, os resultados demonstraram resultados surpreendentes, com elevados percentuais de participantes classificados como ativos e muito ativos em comparação a outros estudos realizados durante a pandemia (Crochemore-Silva et al., 2020; Malta et al., 2020). Talvez este resultado esteja associado a um viés no recrutamento da amostra, uma vez que o formulário do estudo foi compartilhado, principalmente em grupos em que os membros eram estudantes ou docentes da área da Educação Física. No entanto, este viés pouco compromete o objetivo geral do estudo, uma vez que não se pretende avaliar a prevalência deste fator, mas a associação da prática de atividade física com a qualidade do sono.

Em relação ao nível de atividade física relacionado ao sexo, notou-se uma maior proporção de indivíduos do sexo masculino classificados como muito ativo do que as participantes do sexo feminino. Achados similares são mostrados em estudos populacionais anteriores à pandemia (Casas et al., 2018; Mielke et al., 2015), assim como durante o período em que o distanciamento social (Malta et al., 2020). Por exemplo, o estudo de Crochemore-Silva et al. (2020) encontrou diferenças significativas entre os sexos, no qual 37,1\% dos participantes do sexo masculino afirmaram praticar atividade física nesse período, enquanto no sexo feminino o percentual foi de apenas 16,9\%. Além disso, historicamente no Brasil a maior 
responsabilidade pelo trabalho doméstico formal ou não é majoritariamente atribuída às mulheres (Macêdo, 2020). É possível que em decorrência do distanciamento social este fator tenha grande influência no resultado aqui obtido, uma vez que com mais responsabilidades (cozinhar, lavar, cuidar dos filhos, entre outros) o tempo para a prática de atividade física pode ter sido consideravelmente alterado.

Em relação a sexo e qualidade do sono, o estudo não encontrou associação significativa com o sexo. No entanto, os dados são preocupantes, pois quase $80 \%$ apresentam algum nível de dificuldade do sono. Em comparação a alguns estudos prévios, com base populacional realizados anteriores à pandemia (Barros et al., 2019; Zanuto et al., 2015), a prevalência de dificuldade do sono no presente estudo está bastante elevada. Por outro lado, o estudo de Pinto et al. (2020), realizado durante a pandemia, evidenciou que 69,6\% dos participantes apresentou alguma dificuldade de dormir, se aproximando dos achados do presente estudo. Neste sentido, parece que a população passou a ter maiores problemas de sono durante a pandemia, como é evidenciado por Barros et al. (2020) em um estudo populacional com 45.161 participantes das diferentes regiões do Brasil. Foi demonstrado no estudo que 43,5\% dos participantes que não eram acometidos com problemas de sono passaram a ter e dos que já que possuíam $48 \%$ tiveram um aumento dessa condição. Uma pesquisa realizada na Itália com estudantes universitários e funcionários administrativos mostrou que ambos apresentaram declínio significativo na qualidade do sono durante o período da pandemia da COVID-19, no qual as mudanças na qualidade do sono diferenciaram-se dependendo do cronotipo, podendo estarem relacionadas a falta de um cronograma rígido durante o período de distanciamento social (Marelli et al., 2020).

Quando relacionamos a atividade física à qualidade do sono, os resultados do odds ratio demonstraram uma maior chance de indivíduos ativos/muito ativos de apresentarem melhor qualidade do sono em comparação a sedentários/insuficientemente ativos. Embora esta seja uma relação já suportada na literatura (Mello et al., 2005; Ropke et al., 2018), no contexto de pandemia as informações ainda são incipientes. Um dos estudos que investigaram esta associação durante a pandemia comparou praticantes e não praticantes de exercício físico de forma regular, na cidade chinesa de Wuhan. O estudo apontou a falta da prática de atividade física como uma das variáveis relacionadas aos distúrbios do sono (Fu et al., 2020) corroborando com o presente estudo.

Levando em consideração estes resultados, parece que a relação existente entre atividade física e qualidade do sono não são impactadas devido no contexto da pandemia. No entanto, o presente estudo vai além quando se propõe a analisar um desfecho secundário, que é analisar o efeito de diferentes níveis de atividade física na qualidade do sono. Foi observado que ser muito ativo durante a pandemia proporciona uma melhor qualidade do sono em comparação aos demais níveis de atividades física. Além disso, os participantes ativos apresentaram médias do somatório do questionário de dificuldades do sono similares aos sedentários e insuficientemente ativos.

Estes achados nos permitem supor que pessoas que praticam atividade física por um tempo mais prolongado e/ou de maior intensidade apresentam menor dificuldade relacionada ao sono no contexto pandêmico. Diante de todas as incertezas, ansiedade e stress proporcionados pela pandemia, parece que a melhor qualidade do sono pode estar associada a maior sensação de fadiga proporcionada pelo exercício, o que vai ao encontro da hipótese da conservação de energia. Esta hipótese tenta explicar a relação entre exercício físico e sono, defendendo que o aumento do dispêndio energético por meio do exercício físico eleva a necessidade de sono com o objetivo de reestabelecer o balanço energético (Driver \& Taylor, 2000; Mello et al., 2005). Embora exista um nível ótimo de duração e intensidade para que o exercício proporcione benefícios para o sono (Forcina, Mello, \& Tufik, 2001), alguns estudos apontam uma relação entre duração/intensidade do exercício e sono. Shapiro et al. (1975) há várias décadas demonstraram um aumento no sono de ondas leves conforme se aumentava a duração e carga do exercício. É durante o sono de ondas leves que ocorre a restauração física, sendo ela a fase mais profunda do sono (Mello et al., 2005).

Outro indício que pode fornecer suporte ao resultado encontrado é o estudo de Oliveira Silva, Santos, Ritti-Dias e 
Diniz (2018) que comparou o efeito da atividade física e do exercício físico na percepção da qualidade do sono de adolescentes. No referido estudo, ao contrário do exercício físico, a atividade física não foi associada à melhor qualidade do sono. Os autores justificaram este resultado pelo fato de o exercício físico ser caracterizado como uma atividade física mais sistematizada, com objetivo de manter ou melhorar o condicionamento físico, em que o dispêndio energético é maior em comparação à atividade física. Embora o presente estudo tenha investigado o nível de atividade física de forma ampla, com base na análise das correlações que apontou relação significativa apenas para o tempo gasto em atividades vigorosas e somatório do MSQ, podemos supor que boa parte das atividades vigorosas praticadas pelos participantes do estudo podem ser caraterizadas como exercícios físicos, apresentando um dispêndio energético relevante ao ponto de influenciar na qualidade do sono.

\section{Conclusão}

O presente estudo buscou verificar se a prática de atividade física poderia contribuir para a qualidade do sono durante o período de distanciamento social. Foi observado que a atividade física está associada à qualidade do sono. No entanto, esta relação depende do nível da atividade física praticada, com as pessoas que apresentam nível muito ativo tendo melhor qualidade do sono. As atividades físicas vigorosas, aquelas caracterizadas por uma maior duração e intensidade proporcionam uma menor percepção de dificuldade do sono.

A importância da atividade física para a melhoria da qualidade do sono já é relatada e discutida na literatura há algum tempo. No entanto, para o contexto de pandemia o presente estudo fornece insights interessantes para aquelas pessoas que procuram na atividade física uma forma de manter a saúde durante este período. Embora o principal resultado demonstre a necessidade de realização de atividades físicas mais vigorosas, recomenda-se que os praticantes procurem sempre a orientação de profissionais de Educação Física, uma vez que estes profissionais podem prescrever programas de treinamento com a intensidade e volume adequados ao nível de condicionamento físico e objetivos do praticante.

Como limitação, o estudo não investigou outras variáveis que podem interferir nos resultados relacionados à qualidade do sono, como dieta, nível de consumo alcoólico, padrão socioeconômico, entre outros fatores. Recomenda-se que estas variáveis possam ser investigadas em futuros estudos. Além disso, também é interessante a utilização de instrumentos que possam diferenciar atividade física e exercício físico na associação com a qualidade do sono no contexto de pandemia. Pesquisas que investiguem a influência da atividade física em fatores relacionados à saúde e bem estar são de grande relevância no contexto atual. Afinal, após quase um ano do início da pandemia, os índices de contágio e mortalidade da doença continuam a crescer.

\section{Agradecimentos}

Agradecemos aos participantes da pesquisa que cederam um pouco de tempo para contribuir com a construção deste estudo.

\section{Referências}

ABSONO. (2020). Sono e saúde mental, juntos. Sono, 21(1).

Ammar, A., Brach, M., Trabelsi, K., Chtourou, H., Boukhris, O., Masmoudi, L., \& Hammouda, O. (2020). Effects of COVID-19 Home Confinement on Eating Behaviour and Physical Activity: Results of the ECLB-COVID19 International Online Survey. Nutrients, $12,1583-1596$.

Barros, M. B. A., Lima, M. G., Ceolim, M. F., Zancanella, E., \& Caardoso, T. A. M. 0. (2019). Qualidade do sono, saúde e bem-estar em estudo de base populacional. Scopus, 53, 1-12. 
Barros, M. B. de A., Lima, M. G., Malta, D. C., Szwarcwald, C. L., Azevedo, R. C. S. de, Romero, D., \&Gracie, R. (2020). Report on sadness/depression, nervousness/anxiety and sleep problems in the Brazilian adult population during the COVID-19 pandemic. Epidemiologia e Servicos de Saude : Revista Do Sistema Unico de Saude Do Brasil, 29(4), e2020427. https://doi.org/10.1590/s1679-49742020000400018

Bezerra, A. C. V, da Silva, C. E. M., Soares, F. R. G., \& da Silva, J. A. M. (2020). Factors associated with people's behavior in social isolation during the covid-19 pandemic. Ciencia e Saude Coletiva, 25(suppl. 1), 2411-2421. https://doi.org/10.1590/1413-81232020256.1.10792020

Casas, R. C. R. Las, Bernal, R. T. I., Jorge, A. de O., Melo, E. M. de, \& Malta, D. C. (2018). Fatores associados à prática de Atividade Física na população brasileira - Vigitel 2013. Saúde Em Debate, 42(spe4), 134-144. https://doi.org/10.1590/0103-11042018s410

Costa, C. L. A., Costa, T. M., Barbosa Filho, V. C., Bandeira, P. F. R., \& Siqueira, R. C. L. (2020). Influência do distanciamento social no nível de atividade física durante a pandemia do COVID-19. Revista Brasileira de Atividade Física \& Saúde, 25(September), 1-6. https://doi.org/10.12820/rbafs.25e0123

Crochemore-Silva, I., Knuth, A. G., Wendt, A., Nunes, B. P., Hallal, P. C., Santos, L. P., \& Pellegrini, D. da C. P. (2020). Physical activity during the COVID19 pandemic: A population-based cross-sectional study in a city of south Brazil. Ciencia e Saude Coletiva, 25(11), 4249-4258. https://doi.org/10.1590/1413812320202511.29072020

Driver, H. S., \& Taylor, S. R. (2000). Exercise and sleep. Sleep Medicine Reviews, 4(4), 387-402. https://doi.org/10.1053/smrv.2000.0110

Falavigna, A., Bezerra, M. L. B., Teles, A. R., Kleber, F. D., Velho, M. C., Da Silva, R. C., \& Medina, M. F. L. (2011). Consistency and reliability of the Brazilian Portuguese version of the Mini-Sleep Questionnaire in undergraduate students. Sleep and Breathing, 15(3), 351-355. https://doi.org/10.1007/s11325010-0392-X

Faro, A., Bahiano, M. de A., Nakano, T. de C., Reis, C., Silva, B. F. P. da, \& Vitti, L. S. (2020). COVID-19 e saúde mental: a emergência do cuidado. Estudos de Psicologia (Campinas), 37(e200074), 1-14. https://doi.org/10.1590/1982-0275202037e200074

Forcina, P. J. F., Mello, M. T., \& Tufik, S. (2001). Exercício e sono. Revista Brasileira de Medicina Do Esporte, 7(1), 28-36.

Fu, W., Wang, C., Zou, L., Guo, Y., Lu, Z., Yan, S., \& Mao, J. (2020). Psychological health, sleep quality, and coping styles to stress facing the COVID-19 in Wuhan, China. Translational Psychiatry, 10(1), 1-9. https://doi.org/10.1038/s41398-020-00913-3

Giustino, V., Parroco, A. M., Gennaro, A., Musumeci, G., Palma, A., \& Battaglia, G. (2020). Physical activity levels and related energy expenditure during COVID-19 quarantine among the sicilian active population: A cross-sectional online survey study. Sustainability (Switzerland), 12(11). https://doi.org/10.3390/su12114356

In Loco. (2020, December 24). Índice de Isolamento Social. https://mapabrasileirodacovid.inloco.com.br/pt/

Khan, M. K. A., Chu, Y. L., Kirk, S. F. L., \& Veugelers, P. J. (2015). Are sleep duration and sleep quality associated with diet quality, physical activity, and body weight status? A population-based study of Canadian children. Canadian Journal of Public Health, 106(5), e277-e282. https://doi.org/10.17269/CJPH.106.4892

Macêdo, S. (2020). Ser mulher trabalhadora e mãe no Contexto de Pandemia COVID-19: Tecendo sentidos. Revista Do NUFEN: Phenomenology and Interdisciplinarity, 12(2), 187-204.

Machado, A. K. F. (2017). Problemas de sono em população rural do sul do brasil: um estudo de base populacional. Universidade Federal de Pelotas.

Malta, D. C., Szwarcwald, C. L., Barros, M. B. de A., Gomes, C. S., Machado, Í. E., Souza Júnior, P. R. B. de, ... Gracie, R. (2020). A pandemia da COVID19 e as mudanças no estilo de vida dos brasileiros adultos: um estudo transversal, 2020. Epidemiologia e Serviços de Saúde, 29 (4), e2020407. https://doi.org/10.1590/S1679-49742020000400026

Marelli, S., Castelnuovo, A., Somma, A., Castronovo, V., Mombelli, S., Bottoni, D., \& Ferini-Strambi, L. (2020). Impact of COVID-19 lockdown on sleep quality in university students and administration staff. Journal of Neurology, 267(7). https://doi.org/10.1007/s00415-020-10056-6

Matias, T., Dominski, F. H., \& Marks, D. F. (2020). Human needs in COVID-19 isolation. Journal of Health Psychology, 25(7), 871-882. https://doi.org/10.1177/1359105320925149

Matsudo, S., Araújo, T., Matsudo, V., Andrade, D., Andrade, E., Oliveira, L. C., \& Braggion, G. (2001). Questionário Internacional De Atividade Física (IPAQ): Estudo de validade e reprodutibilidade no Brasil. Revista Brasileira de Atividade Física \& Saúde, 6(2), 5-18. https://doi.org/10.12820/rbafs.v.6n2p518

Mello, M. T. De, Boscolo, R. A., Esteves, A. M., \& Tufik, S. (2005). O exerccio fsico e os aspectos psicobiolgicos. Revista Brasileira de Medicina Do Esporte, 11(3), 203-207.

Mielke, G. I., Malta, D. C., de Sá, G. B. A. R., Reis, R. S., \& Hallal, P. C. (2015). Diferenças regionais e fatores associados à prática de atividade física no lazer no Brasil: Resultados da pesquisa nacional de saúde-2013. Revista Brasileira de Epidemiologia, 18, 158-169. https://doi.org/10.1590/19805497201500060014

Monteiro, B. M. M., Santos Neto, C. N., \& Souza, J. C. R. P. de. (2020). Sono e cronotipo em estudantes universitários na pandemia da COVID-19. Research, Society and Development, 9(9), e632997688. https://doi.org/10.33448/rsd-v9i9.7688.

Nyenhuis, S. M., Greiwe, J., Zeiger, J. S., Nanda, A., \& Cooke, A. C. (2020). Since January 2020 Elsevier has created a COVID-19 resource centre with free information in English and Mandarin on the novel coronavirus COVID- 19. The COVID-19 resource centre is hosted on Elsevier Connect, the company' s public news and information. The Journal of Allergy and Clinical Immunology: In Practice, 8(7), 2152-2155. 
Research, Society and Development, v. 10, n. 1, e8610111471, 2021

(CC BY 4.0) | ISSN 2525-3409 | DOI: http://dx.doi.org/10.33448/rsd-v10i1.11471

Oliveira, L. M. F. T., Silva, A. O., Santos, M. A. M., Ritti-Dias, R. M., \& Diniz, P. R. B. (2018). Exercise or physical activity: Which is more strongly associated with the perception of sleep quality by adolescents? Revista Paulista de Pediatria, 36(3), 322-328. https://doi.org/10.1590/1984.0462/;2018;36;3;00014

Ornell, F., Schuch, J. B., Sordi, A., \& Kessler, F. H. P. (2020). "Pandemic fear"' and COVID-19: mental health burden and strategies. Brazilian Journal of Psychiatry, 42(3), 232-235. https://doi.org/10.1590/1516

Pascarella, G., Strumia, A., Piliego, C., Bruno, F., Del Buono, R., Costa, F., \& Agrò, F. E. (2020). COVID-19 diagnosis and management: a comprehensive review. Journal of Internal Medicine, 288(2), 192-206. https://doi.org/10.1111/joim.13091

Pinto, J., van Zeller, M., Amorim, P., Pimentel, A., Dantas, P., Eusébio, E., ... Drummond, M. (2020). Sleep quality in times of Covid-19 pandemic. Sleep Medicine, 74, 81-85. https://doi.org/10.1016/j.sleep.2020.07.012

Ropke, L. M., Souza, A. G., Bertoz, A. P. de M., Adriazola, M. M., Ortolan, E. V. P., Martins, R. H., \& Weber, S. A. T. (2018). Efeito da atividade física na qualidade do sono e qualidade de vida: revisão sistematizada. Archives of Health Investigation, 6(12), 561-566. https://doi.org/10.21270/archi.v6i12.2258

Shapiro, C. M., Griesel, R. D., Bartel, P. R., \& Jooste, P. L. (1975). Sleep patterns after graded exercise. Journal of Applied Physiology, 39(2), 187-190. https://doi.org/10.1152/jappl.1975.39.2.187

Silveira, A. L. B., Carvalho, L. M., Seara, F. A. C., \& Olivares, E. L. (2020). Wear a mask to reduce COVID-19 transmission while exercising at the gym: belief or evidence-based? Research, Society and Development, 9(10), e8499109259. https://doi.org/10.33448/rsd-v9i10.9259.

Thomas, J. R. E., Nelson, J. K., \& Silverman, S. J. (2012). Métodos de Pesquisa em Atividade Física (6th ed.). Artimed.

Wilder-Smith, A., \& Freedman, D. O. (2020). Isolation, quarantine, social distancing and community containment: Pivotal role for old-style public health measures in the novel coronavirus (2019-nCoV) outbreak. Journal of Travel Medicine, 27(2), 1-4. https://doi.org/10.1093/jtm/taaa020

Wu, X., Tao, S., Zhang, Y., Zhang, S., \& Tao, F. (2015). Low physical activity and high screen time can increase the risks of mental health problems and poor sleep quality among Chinese college students. PLoS ONE, 10(3), 1-10. https://doi.org/10.1371/journal.pone.0119607

Zanuto, A. C. E., De Limai, C. S. M., De Araújo, G. R., Da Silva, P. E., Anzolin, C. C., Araujo, Y. C. M., \& Fernandes, R. A. (2015). Distúrbios do sono em adultos de uma cidade do estado de São Paulo. Revista Brasileira de Epidemiologia, 18(1), 42-53. https://doi.org/10.1590/1980-5497201500010004 